\title{
Sleep Disorder and Long-Term Mortality Among Sepsis Survivors: A Nationwide Cohort Study in South Korea
}

\author{
In-Ae Song ${ }^{1, *}$ \\ Hye Yoon Park ${ }^{2,3, *}$ \\ Tak Kyu Oh iD ' \\ 'Department of Anesthesiology and Pain \\ Medicine, Seoul National University \\ Bundang Hospital, Seongnam, South \\ Korea; ${ }^{2}$ Department of Psychiatry, Seoul \\ National University Hospital, Seoul, \\ South Korea; ${ }^{3}$ Department of Psychiatry, \\ College of Medicine, Seoul National \\ University, Seoul, South Korea \\ *These authors contributed equally to \\ this work
}

Correspondence: Tak Kyu Oh

Department of Anesthesiology and Pain Medicine, Seoul National University

Bundang Hospital, Gumi-ro 173 Beon-gil, Bundang-gu, Seongnam, I3620, South

Korea

Tel +82-31-787-7499

Email airohtak@hotmail.com
Background: We aimed to investigate the association of sleep disorder diagnosis among sepsis survivors with 5-year all-cause mortality.

Methods: Using the National Health Insurance Service (NHIS) database of South Korea, we included adult sepsis survivors who were primarily diagnosed with sepsis between 2011 and 2014 and survived for more than one year after diagnosis. The diagnosis of sleep disorders was evaluated using the International Classification of Diseases, 10th revision codes of G47* in the NHIS database.

Results: In total, 45,826 survivors of sepsis were included in this analysis. Among the sepsis survivors, 2935 (6.4\%) were newly diagnosed with a sleep disorder within 1 year after the date of sepsis diagnosis, while 7938 (17.3\%) were already diagnosed with sleep disorder before the date of sepsis diagnosis. In the multivariable Cox regression, the risk of 5-year allcause mortality in the pre- and post-sepsis sleep disorder groups was 1.19-fold (hazard ratio: 1.19, 95\% confidence interval: $1.14-1.24 ; P<0.001$ ) and 1.79 -fold (hazard ratio: $1.79,95 \%$ confidence interval: $1.70-1.89 ; P<0.001$ ) higher than that of the control group.

Conclusion: A $6.4 \%$ of sepsis survivors in South Korea were newly diagnosed with a sleep disorder within 1 year of sepsis diagnosis. Although both pre- and post-sepsis sleep disorders were associated with a higher 5-year all-cause mortality rate, the risk of the 5-year all-cause mortality in the post-sepsis sleep disorder group was higher than that in the pre-sepsis sleep disorder group.

Keywords: critical care, intensive care units, sepsis, survivors, sleep wake disorders

\section{Introduction}

Sepsis is characterized as a life-threatening condition attributable to a dysregulated host immune response to infection. ${ }^{1}$ In the United States, it was reported that $6 \%$ of hospitalized adults had sepsis. ${ }^{2}$ However, in-hospital mortality declined from $24.1 \%$ to $14.8 \%$ from 2010 to $2015^{3,4}$ due to many efforts made to treat sepsis. ${ }^{5}$ Recent cohort studies have reported that the improved survival for these patients was due to advancements in medical management and treatment. ${ }^{6-8}$ Therefore, quality of life and return to work have emerged as important issues for survivors of sepsis.

Sleep is considered a physiological necessity, and sleep disorders are a common age-related problem that can lead to distress and discomfort, impaired daytime functioning, and serious complications. ${ }^{9}$ Approximately $7.1-9.8 \%$ of the population in the United States have been diagnosed with a sleep disorder. ${ }^{10,11}$ These patients with sleep disorders reportedly have a 1.5 -fold higher mortality rate than the 
general population with no sleep disorders. ${ }^{11}$ Therefore, the prevention and treatment of sleep disorders have become important public health issues. A previous study reported that critically ill patients often suffer from sleep disturbances due to the development of delirium and the use of sedative agents during sepsis treatment. ${ }^{12}$ Sleep disturbances manifest in the treatment during hospitalization and are a significant sequalae after discharge from the hospital among survivors. ${ }^{13}$ Rundshage et al reported that sedated and ventilated critically ill patients discharged from intensive care units (ICUs) suffered from nightmares $(9.3 \%)$ and hallucinations $(6.6 \%) .{ }^{14}$ In addition, it was reported that post-traumatic stress disorder (PTSD) related to ICU stays was associated with the development of amnesia and memory impairment after ICU discharge. ${ }^{15}$ From these perspectives, sleep disorder development after sepsis can significantly affect the quality of daily life among sepsis survivors. However, the prevalence of sleep disorder development among sepsis survivors after sepsis treatment has not yet been studied, and its association with long-term prognosis remains controversial.

Therefore, we aimed to investigate the prevalence of sleep disorder diagnosis among sepsis survivors and identify its association with 5-year all-cause mortality. We hypothesized that pre- and post-sepsis sleep disorders were associated with a higher risk of 5-year all-cause mortality among sepsis survivors.

\section{Materials and Methods}

\section{Ethical Statement and Data Source}

This population-based cohort study was conducted according to the Reporting of Observational Studies in Epidemiology guidelines. The study protocol was approved by the Institutional Review Board of Seoul National University Bundang Hospital (X-1912-580-902) and the Health Insurance Review and Assessment Service (NHIS-2020-1-095). Informed consent was waived because data analyses were performed retrospectively using anonymized data extracted by an independent medical record technician at the South Korean National Health Insurance Service (NHIS) center unaffiliated with this study.

\section{Study Population}

We initially screened all adult patients ( $\geq 18$ years of age) admitted to any hospital in South Korea between 2011 and 2014 for the main diagnosis of sepsis (A40, A41) or septic shock (R65.2), according to the International Classification of Diseases, 10th revision (ICD-10) codes. The NHIS database contains all newly registered disease diagnoses made during hospitalization, and the diagnosis registered as the primary morbidity of treatment is then classified as the main diagnosis. That is, if a patient initially admitted with a diagnosis of urinary tract infection was diagnosed as sepsis during treatment, the main diagnosis of the patient would be classified as sepsis, by NHIS. If a patient was admitted to any hospital for sepsis treatment two times or more during the study period, only the first episode was included in the study. Among all patients who were mainly diagnosed with sepsis during hospitalization, those identified as sepsis survivors (ie, those who had survived for over 365 days after sepsis diagnosis) were eligible for inclusion in this study.

\section{Sleep Disorder as a Main Independent Variable}

The diagnosis of sleep disorders was evaluated using the ICD-10 codes of G47* (G47.0: primary insomnia, and G47.1-9) in the NHIS database. The sepsis survivors were classified into the following three groups: 1) Presepsis sleep disorder group, diagnosed with sleep disorder before the date of diagnosis of sepsis; 2) post-sepsis sleep disorder group, with no prior history of sleep disorders, but newly diagnosed with a sleep disorder within 1 year of sepsis diagnosis; and 3) control group, not diagnosed with a sleep disorder before or after the date of diagnosis of sepsis.

\section{Endpoint}

The primary endpoint of this study was 5-year all-cause mortality (additional 4-year all-cause mortality) among survivors of sepsis, defined as any death of survivors of sepsis after the diagnosis of sepsis within 5 years. The survival times of sepsis survivors were calculated from the date of sepsis diagnosis to the date of death or April 1, 2020 because the mortality dates were extracted until December 31, 2019.

\section{Covariates}

Data extracted as confounders included demographic characteristics (age and sex) and place of residence at the time of diagnosis (Seoul, metropolitan city, other areas, and unknown). The annual income level of sepsis survivors in terms of the Korean currency (Won) was extracted and 
classified into five groups based on quartile ratio $(\mathrm{Q} 1$ : lowest, Q2, Q3, Q4: highest, and unknown group). To reflect the severity of sepsis patients, endotracheal intubation, continuous vasopressor infusion, mechanical ventilatory support, continuous renal replacement (CRRT) use, and extracorporeal membrane oxygenation (ECMO) use during sepsis treatment were collected as confounders. The vasopressor infusion includes the infusion of epinephrine, norepinephrine, vasopressin, dopamine, and dobutamine. Data on the total sepsis treatment duration was categorized into four groups $(1-7,8-15,16-30$, and $>30$ days). Information regarding how the sepsis survivor was followed up after hospital discharge was divided into three groups as follows: 1) follow-up in the same hospital, 2) referred to another hospital for admission, and 3) outpatient clinic follow-up. To reflect the comorbid status at the diagnosis of sepsis, we used the underlying disability before sepsis and the Charlson comorbidity index. The ICD-10 codes calculated for determining the Charlson score before sepsis treatment are presented in Appendix 1, and the ICD-10 codes for pre-sepsis were extracted from within 1 year prior to sepsis diagnosis. In addition, underlying depression (ICD-10 codes: F32, F33, F34.1) and PTSD (ICD-10 codes: F43*) were extracted and included as covariates for adjustment because sleep disorder is known to be often accompanied by PTSD or depression. ${ }^{16,17}$ Regarding sleeping pills, the prescription information of benzodiazepines and Z-drugs (zaleplon, zopiclone, eszopiclone, and zolpidem) was extracted and used as covariates for adjustment. If a sepsis survivor was prescribed the drugs for over 30 days, he/she was defined as a benzodiazepine or Z-drug user.

\section{Statistical Analysis}

Baseline characteristics of sepsis survivors are presented as means with standard deviations for continuous variables and numbers with percentages for categorical variables. First, we compared the baseline characteristics between the three groups using a one-way ANOVA test for continuous variables and the chi-square test for categorical variables. Second, we constructed a multivariable Cox regression model to investigate whether sleep disorder before or after sepsis affected the risk of 5-year all-cause mortality. In the multivariable model, the risk of 5-year all-cause mortality in the pre-sepsis sleep disorder group and post-sepsis sleep disorder group was compared to that of the control group. All covariates were included in the multivariable model for adjustment. Third, a separate multivariable Cox regression model was constructed to examine the risk of 5-year all-cause mortality in pre- and post-sepsis insomnia and the other sleep disorder group compared to that of the control group. Fourth, we performed sensitivity analyses of the multivariable Cox regression model for 5-year all-cause mortality according to sleep disorder with depression or PTSD and sleep disorder with benzodiazepines or Z-drug use. Sleep disorder is commonly accompanied by PTSD or depression, ${ }^{16,17}$ and benzodiazepines or Z-drug use might affect longterm mortality in patients with sleep disorders. ${ }^{18}$ Therefore, sensitivity analyses are needed to confirm the impact of PTSD or depression and benzodiazepine or Z-drug use on the outcomes in this study. Multivariable Cox regression modeling was used for the subgroup analyses using the same method as the main analysis. The results of the Cox model were presented as hazard ratios (HRs) with 95\% confidence intervals (CIs) and confirmed using log-log plots that the central assumption of the multivariable Cox regression model was satisfied. $\mathrm{C}$-statistics were used to identify the C-index of the multivariable Cox regression model, and there was no collinearity between the variables in the model (variance inflation factor $<2.0$ ). All statistical analyses were performed using $\mathrm{R}$ software (version 4.0.3 with R packages, the R Project for Statistical Computing, Vienna, Austria), and values of $P<0.05$ were considered statistically significant.

\section{Results \\ Study Population}

From 2011 to 2014, 149,278 patients were diagnosed with sepsis and admitted to hospitals 284,556 times for treatment. Among these patients, 93,722 (62.8\%) were alive for more than one year after sepsis diagnosis; accordingly, they were defined as survivors of sepsis. Subsequently, we excluded 47,896 pediatric patients $(<18$ years old) from the analysis. Finally, a total of 45,826 survivors of sepsis, alive for more than one year after sepsis diagnosis, were included in this analysis (Figure 1). The baseline characteristics of the sepsis survivors in this study are presented in Table 1. Among the sepsis survivors, 2935 (6.4\%) were newly diagnosed with a sleep disorder within 1 year after the date of sepsis diagnosis and defined as the post-sepsis sleep disorder group, while 7938 (17.3\%) were already diagnosed with sleep disorder before the date of sepsis diagnosis and defined as the pre-sepsis sleep disorder group. The prevalence of insomnia was 11.2\% (5120/ 


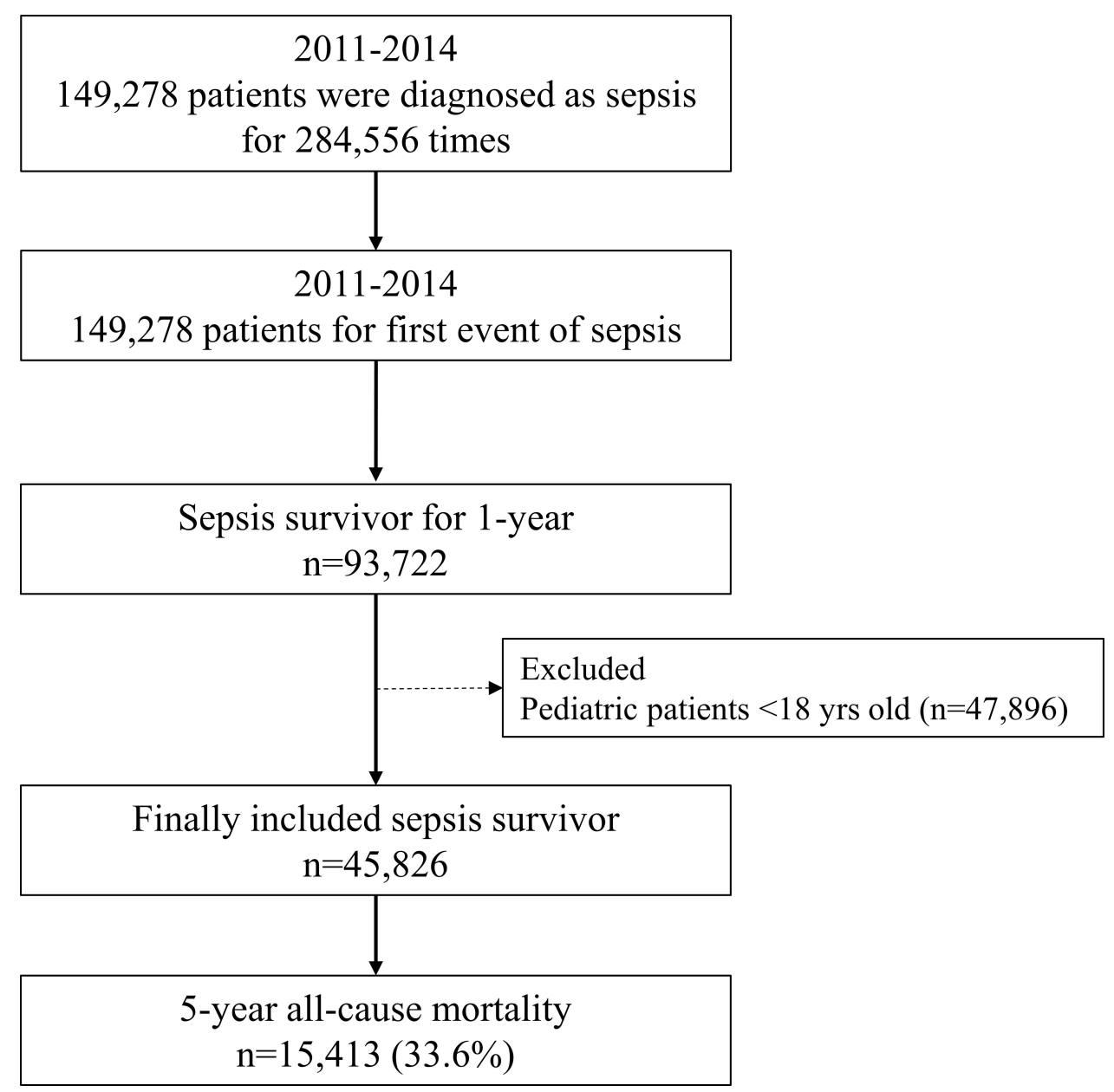

Figure I Flow chart depicting patient selection.

$45,826)$ and $4.3 \%(1988 / 45,826)$ in pre- and post-sepsis sleep disorder groups, respectively.

\section{Five-Year All-Cause Mortality}

Table 2 shows the results of the comparison of characteristics between the three groups among sepsis survivors. The 5 -year all-cause mortality rate was $47.0 \%(6536 / 45,826)$ in the pre-sepsis sleep disorder group, $40.5 \%(1678 / 45,826)$ in the post-sepsis sleep disorder group, and 25.9\% (7199/ 45,826 ) in the control group. In the multivariable Cox regression model 1 (Table 3), the risk of 5-year all-cause mortality in the pre- and post-sepsis sleep disorder groups was 1.19-fold (HR:1.19, 95\% CI: 1.15-1.24; $P<0.001$ ) and 1.79-fold (HR:1.79, 95\% CI: 1.70-1.89; $P<0.001$ ) higher than that of the control group. In the multivariable Cox regression model 2, the pre-sepsis insomnia group and other sleep disorder group showed a 1.15-fold (HR:1.15, 95\% CI: $1.10-1.23 ; P<0.001)$ and a 1.05-fold (HR:1.05, 95\% CI: $1.03-1.06 ; P=0.014)$ higher 5-year all-cause mortality risk than the control group, respectively. In addition, the post-sepsis insomnia group and other sleep disorder group showed a 1.89-fold (HR:1.89, 95\% CI: 1.85-1.94; $P<0.001$ ) and a 1.44-fold (HR:1.44, 95\% CI: 1.39-1.48; $P<0.001)$ higher 5-year all-cause mortality risk than the control group, respectively. The $\mathrm{C}$-index of the multivariable model was 0.82 (95\% CI: 0.81-0.82). The survival plot derived from the multivariable Cox regression model showed a similar trend, as shown in Appendix 2.

\section{Sensitivity Analyses}

Table 4 shows the results of sensitivity analyses of the multivariable Cox regression model for 5-year all-cause mortality according to sleep disorder with depression or PTSD and sleep disorder with benzodiazepines or Z-drug use. Compared to the control group (multivariable model 1), the pre-sepsis sleep disorder group was associated with 1.19fold (HR: 1.19, 95\% CI: 1.13-1.25; $P<0.001)$ and 1.21-fold (HR: $1.21,95 \%$ CI: 1.13-1.29; $P<0.001)$ higher 5-year all- 
Table I Baseline Characteristics of Total Sepsis Survivor from 2011 to $2014(n=45,826)$

\begin{tabular}{|c|c|c|}
\hline Variable & $\mathbf{N}(\%)$ & Mean (SD) \\
\hline Age, year & & $62.9(19.3)$ \\
\hline Gender, Male & $19,055(41.6)$ & \\
\hline \multicolumn{3}{|l|}{ Residence at diagnosis of sepsis } \\
\hline Seoul (Capital city) & $10,859(23.7)$ & \\
\hline Other metropolitan city & $8296(18.1)$ & \\
\hline Other area & $26,671(58.2)$ & \\
\hline \multicolumn{3}{|l|}{ Income level at diagnosis of sepsis } \\
\hline QI (Lowest) & $6993(15.3)$ & \\
\hline Q2 & $6793(14.8)$ & \\
\hline Q3 & 9111 (19.9) & \\
\hline Q4 (Highest) & $15,408(33.6)$ & \\
\hline Unknown & $7521(16.4)$ & \\
\hline Underlying disability before sepsis & II,843 (25.8) & \\
\hline Charlson comorbidity index before sepsis & & $4.8(3.7)$ \\
\hline \multicolumn{3}{|l|}{ Total treatment duration for sepsis, day } \\
\hline $1-7$ & $22,893(50.0)$ & \\
\hline $8-15$ & $10,655(23.3)$ & \\
\hline $16-30$ & $8258(18.0)$ & \\
\hline$>30$ & $4020(8.8)$ & \\
\hline \multicolumn{3}{|l|}{ Treatment of sepsis during hospitalization } \\
\hline Mechanical ventilator use & $2295(5.0)$ & \\
\hline Endotracheal intubation & $|77|(3.9)$ & \\
\hline CRRT use & $491(1.1)$ & \\
\hline Vaspressor use & $3255(7.1)$ & \\
\hline ECMO use & $12(0.0)$ & \\
\hline \multicolumn{3}{|l|}{ Follow up after discharge } \\
\hline Follow up in the same hospital & $29,438(64.2)$ & \\
\hline Referred to other hospital for admission & $2039(4.4)$ & \\
\hline Outpatient clinic follow up & $\mid 4,349(3 \mid .3)$ & \\
\hline \multicolumn{3}{|l|}{ Year of diagnosis of sepsis } \\
\hline 2011 & $10,205(22.3)$ & \\
\hline 2012 & $10,958(23.9)$ & \\
\hline 2013 & II,436 (25.0) & \\
\hline 2014 & 13,227 (28.9) & \\
\hline Underlying PTSD & $72(0.2)$ & \\
\hline Underlying depression & $|0,73|(23.4)$ & \\
\hline $\mathrm{BDZ}$ or Z-drug user & $4191(9.1)$ & \\
\hline \multicolumn{3}{|l|}{ Sleep disorder before and after sepsis } \\
\hline Control group & $34.953(76.3)$ & \\
\hline Pre-sepsis sleep disorder group & $7938(17.3)$ & \\
\hline Insomnia & $5120(11.2)$ & \\
\hline Other sleep disorder & $2818(6.1)$ & \\
\hline Post-sepsis sleep disorder group & $2935(6.4)$ & \\
\hline Insomnia & $1988(4.3)$ & \\
\hline Other sleep disorder & $947(2.1)$ & \\
\hline
\end{tabular}

Abbreviations: SD, standard deviation; CRRT, continuous renal replacement therapy; ECMO, extracorporeal membrane oxygenation; PTSD, post-traumatic stress disorder; BDZ, benzodiazepine. cause mortality rate without and with PTSD or depression, respectively. The post-sepsis sleep disorder group was associated with 1.67-fold (HR: $1.67,95 \%$ CI: 1.56-1.79; $P<0.001$ ) and 2.01-fold (HR: 2.01, 95\% CI: 1.84-2.19; $P<0.001)$ higher 5-year all-cause mortality rate without and with PTSD or depression, respectively. In addition, compared to the control group (multivariable model 2), the pre-sepsis sleep disorder group was associated with 1.18fold (HR: $1.18,95 \%$ CI: $1.13-1.23 ; P<0.001$ ) and 1.30 -fold (HR: $1.30,95 \%$ CI: $1.18-1.43 ; P<0.001$ ) higher 5-year allcause mortality rate without and with benzodiazepines or Z-drug use, respectively. Furthermore, the post-sepsis sleep disorder group was associated with 1.76-fold (HR: 1.76, 95\% CI: $1.66-1.86 ; P<0.001$ ) and 2.02-fold (HR: 2.02, 95\% CI: $1.77-2.30 ; P<0.001$ ) higher 5-year all-cause mortality rate without and with benzodiazepines or Z-drug use, respectively.

\section{Discussion}

This population-based cohort study showed that $6.4 \%$ (2935 of 34,953) of sepsis survivors were newly diagnosed with a sleep disorder within 1 year after sepsis diagnosis. Additionally, sleep disorders both pre- and post-sepsis were associated with a higher 5-year all-cause mortality rate among sepsis survivors. Interestingly, this association was more evident among patients in the post-sepsis sleep disorder group, sleep disorder group with PTSD or depression, and sleep disorder group with benzodiazepine or Z-drug use. Our results are important because this is the first study reporting the prevalence of sleep disorders among survivors of sepsis and its association with longterm mortality.

In a prospective international survey of 1731 sepsis survivors by Huang et al, ${ }^{19}$ it was reported that many survivors of sepsis suffered from sleep disturbances. However, the exact prevalence of sleep disorders was not reported in this study. ${ }^{19}$ In another systemic review, the prevalence of sleep disorders varied from 10-61\% among ICU survivors who were admitted to the hospital for treatment of a critical illness. ${ }^{20}$ Most previous studies have reported prevalence based on questionnaires, ${ }^{19,20}$ while our study reported the prevalence of pre- and postsepsis sleep disorder diagnosis using registered ICD-10 codes in the NHIS database. Therefore, it is possible that only patients diagnosed with a definite sleep disorder were included in our study, and the result should be interpreted carefully. 
Table 2 Comparision of Characteristics Between Three Groups Among Sepsis Survivors

\begin{tabular}{|c|c|c|c|c|}
\hline Variable & $\begin{array}{c}\text { Control Group } \\
n=34,953\end{array}$ & $\begin{array}{c}\text { Pre-Sepsis Sleep Disorder } \\
\qquad \mathrm{n}=7938\end{array}$ & $\begin{array}{c}\text { Post-Sepsis Sleep Disorder } \\
\qquad \mathrm{n}=2935\end{array}$ & $P$-value \\
\hline Age, year & $60.7(19.9)$ & $70.2(14.8)$ & $69.6(15.7)$ & $<0.001$ \\
\hline Gender, Male & 12,485 (44.9) & $4819(34.7)$ & $|75|(42.2)$ & $<0.001$ \\
\hline $\begin{array}{l}\text { Residence at diagnosis of sepsis } \\
\text { Seoul (Capital city) } \\
\text { Other metropolitan city } \\
\text { Other area }\end{array}$ & $\begin{array}{c}7444(26.8) \\
4587(16.5) \\
15,747(56.7)\end{array}$ & $\begin{array}{l}2569(18.5) \\
2903(20.9) \\
8430(60.6)\end{array}$ & $\begin{array}{c}846(20.4) \\
806(19.4) \\
2494(60.2)\end{array}$ & $<0.001$ \\
\hline $\begin{array}{l}\text { Income level at diagnosis of sepsis } \\
\text { Q1 (Lowest) } \\
\text { Q2 } \\
\text { Q3 } \\
\text { Q4 (Highest) } \\
\text { Unknown }\end{array}$ & $\begin{array}{l}4268(15.4) \\
4524(16.3) \\
5958(21.4) \\
9090(32.7) \\
3938(14.2)\end{array}$ & $\begin{array}{l}2060(14.8) \\
1680(12.1) \\
2398(17.2) \\
4963(35.7) \\
2801(20.1)\end{array}$ & $\begin{array}{l}665(16.0) \\
589(14.2) \\
755(18.2) \\
1355(32.7) \\
782(18.9)\end{array}$ & $<0.001$ \\
\hline Underlying disability before sepsis & $5684(20.5)$ & $4919(35.4)$ & $1240(29.9)$ & $<0.001$ \\
\hline $\begin{array}{l}\text { Charlson comorbidity index before } \\
\text { sepsis }\end{array}$ & $4.5(3.7)$ & $6.0(3.6)$ & $6.1(3.9)$ & $<0.001$ \\
\hline \multicolumn{5}{|l|}{$\begin{array}{l}\text { Treatment of sepsis during } \\
\text { hospitalization }\end{array}$} \\
\hline Mechanical ventilator use & $1518(4.3)$ & $573(7.2)$ & $204(7.0)$ & $<0.001$ \\
\hline Endotracheal intubation & $1167(3.3)$ & $456(5.7)$ & $148(5.0)$ & $<0.001$ \\
\hline CRRT use & $344(1.0)$ & $107(1.3)$ & $40(1.4)$ & 0.005 \\
\hline Vaspressor use & $2268(6.5)$ & $764(9.6)$ & $223(7.6)$ & $<0.001$ \\
\hline ECMO use & $6(0.0)$ & $4(0.1)$ & $2(0.1)$ & 0.089 \\
\hline $\begin{array}{l}\text { Total treatment duration for sepsis, day } \\
\qquad \begin{array}{l}1-7 \\
8-15 \\
16-30 \\
>30\end{array}\end{array}$ & $\begin{array}{c}15,952(57.4) \\
5506(19.8) \\
4386(15.8) \\
1934(7.0)\end{array}$ & $\begin{array}{l}5393(38.8) \\
4049(29.1) \\
2930(21.1) \\
1530(11.0)\end{array}$ & $\begin{array}{l}1548(37.3) \\
1100(26.5) \\
942(22.7) \\
556(13.4)\end{array}$ & $<0.001$ \\
\hline $\begin{array}{l}\text { Follow up after discharge } \\
\text { Follow up in the same hospital } \\
\text { Referred to other hospital for } \\
\text { admission } \\
\text { Outpatient clinic follow up }\end{array}$ & $\begin{array}{l}18,724(67.4) \\
1102(4.0) \\
7952(28.6)\end{array}$ & $\begin{array}{c}8394(60.4) \\
687(4.9) \\
4821(34.7)\end{array}$ & $\begin{array}{c}2320(56.0) \\
250(6.0) \\
1576(38.0)\end{array}$ & $<0.001$ \\
\hline Underlying depression or PTSD & $6570(18.8)$ & $3142(39.6)$ & $1057(36.0)$ & $<0.001$ \\
\hline BDZ or Z-drug user & $2135(6.1)$ & $1529(19.3)$ & $503(17.1)$ & $<0.001$ \\
\hline $\begin{array}{l}\text { Year of diagnosis of sepsis } \\
2011 \\
2012 \\
2013 \\
2014\end{array}$ & $\begin{array}{l}6955(25.0) \\
6838(24.6) \\
6594(23.7) \\
7391(26.6)\end{array}$ & $\begin{array}{l}2242(16.1) \\
3115(22.4) \\
3827(27.5) \\
4718(33.9)\end{array}$ & $\begin{array}{l}1008(24.3) \\
1005(24.2) \\
1015(24.5) \\
1118(27.0)\end{array}$ & $<0.001$ \\
\hline 5-year all-cause mortality & $7199(25.9)$ & $6536(47.0)$ & $1678(40.5)$ & $<0.001$ \\
\hline
\end{tabular}

Note: Presented as number with percentage or mean with standard deviation.

Abbreviations: CRRT, continuous renal replacement therapy; ECMO, extracorporeal membrane oxygenation; PTSD, post-traumatic stress disorder; BDZ, benzodiazepine.

Recently, we reported that adult individuals with sleep disorders were associated with a 1.23 -fold higher 5 -year all-cause mortality than adult individuals without sleep disorders among the South Korean population. ${ }^{21}$ In our study, the 5-year all-cause mortality among sepsis survivors with pre- and post-sepsis sleep disorder showed a 1.19-fold and a 1.79-fold increase in the 5-year allcause mortality, respectively. It suggested that the higher 
Table 3 Multivariable Cox Model for 5-Year All-Cause Mortality Among Sepsis Survivor

\begin{tabular}{|c|c|c|}
\hline \multirow[t]{2}{*}{ Variable } & $\begin{array}{l}\text { Multivariable Cox } \\
\text { Model }\end{array}$ & \multirow[t]{2}{*}{ P-value } \\
\hline & HR (95\% Cl) & \\
\hline \multicolumn{3}{|l|}{$\begin{array}{l}\text { Sleep disorder before and after sepsis } \\
\text { (model I) }\end{array}$} \\
\hline Control group & 1 & \\
\hline Pre-sepsis sleep disorder group & $1.19(1.15,1.24)$ & $<0.001$ \\
\hline Post-sepsis sleep disorder group & $1.79(1.70,1.89)$ & $<0.001$ \\
\hline \multicolumn{3}{|l|}{$\begin{array}{l}\text { Sleep disorder before and after sepsis } \\
\text { (model 2) }\end{array}$} \\
\hline Control group & I & \\
\hline Pre-sepsis insomnia group & $1.15(1.10,1.23)$ & $<0.001$ \\
\hline Pre-sepsis other sleep disorder group & $1.05(1.03,1.06)$ & 0.014 \\
\hline Post-sepsis insomnia group & $1.89(1.85,1.94)$ & $<0.001$ \\
\hline Post-sepsis other sleep disorder group & $1.44(1.39,1.48)$ & $<0.001$ \\
\hline Age, year & $1.06(1.06,1.06)$ & $<0.001$ \\
\hline Gender, Male & $1.50(1.45,1.55)$ & $<0.001$ \\
\hline \multicolumn{3}{|l|}{ Residence at diagnosis of sepsis } \\
\hline Seoul (Capital city) & 1 & \\
\hline Other metropolitan city & $1.11(1.05,1.17)$ & $<0.001$ \\
\hline Other area & $1.08(1.03,1.12)$ & 0.001 \\
\hline \multicolumn{3}{|l|}{ Income level at diagnosis of sepsis } \\
\hline QI (Lowest) & 1 & \\
\hline Q2 & $0.97(0.91,1.03)$ & 0.360 \\
\hline Q3 & $0.95(0.90,1.00)$ & 0.069 \\
\hline Q4 (Highest) & $0.93(0.88,0.98)$ & 0.003 \\
\hline Unknown & $1.18(1.11,1.24)$ & $<0.001$ \\
\hline Underlying disability before sepsis & $1.45(1.40,1.07)$ & $<0.001$ \\
\hline $\begin{array}{l}\text { Charlson comorbidity index before sepsis, } \\
\text { point }\end{array}$ & $1.06(1.06,1.07)$ & $<0.001$ \\
\hline \multicolumn{3}{|l|}{ Treatment of sepsis during hospitalization } \\
\hline Mechanical ventilator use & $1.35(1.22,1.51)$ & $<0.001$ \\
\hline Endotracheal intubation & $0.94(0.83,1.05)$ & 0.277 \\
\hline CRRT use & $0.77(0.66,0.89)$ & 0.001 \\
\hline Vaspressor use & $0.89(0.83,0.95)$ & $<0.001$ \\
\hline ECMO use & $1.72(0.64,4.59)$ & 0.283 \\
\hline \multicolumn{3}{|l|}{ Total treatment duration for sepsis, day } \\
\hline $1-7$ & 1 & \\
\hline $8-15$ & $1.46(1.40,1.52)$ & $<0.001$ \\
\hline $16-30$ & $1.36(1.29,1.42)$ & $<0.001$ \\
\hline$>30$ & $1.50(1.41,1.59)$ & $<0.001$ \\
\hline \multicolumn{3}{|l|}{ Follow up after discharge } \\
\hline Follow up in the same hospital & 1 & \\
\hline Referred to other hospital for admission & $0.82(0.76,0.88)$ & $<0.001$ \\
\hline Outpatient clinic follow up & $0.63(0.60,0.65)$ & $<0.001$ \\
\hline Underlying depression or PTSD & $1.00(0.99,1.01)$ & 0.190 \\
\hline $\mathrm{BDZ}$ or Z-drug user & $1.00(0.99,1.00)$ & 0.522 \\
\hline
\end{tabular}

(Continued)
Table 3 (Continued).

\begin{tabular}{|l|c|c|}
\hline Variable & $\begin{array}{c}\text { Multivariable Cox } \\
\text { Model }\end{array}$ & \multirow{2}{*}{ P-value } \\
\cline { 2 - 2 } & HR (95\% Cl) & \\
\hline Year of diagnosis of sepsis & 1 & \\
2011 & $0.88(0.84,0.92)$ & \\
2012 & $0.88(0.84,0.92)$ & $<0.001$ \\
2013 & $0.84(0.80,0.88)$ & $<0.001$ \\
2014 & \\
\hline
\end{tabular}

Notes: C-index: $0.82(0.81,0.82)$. Model fitting information: -2 log likeliwood 308843.0, Chi-square: 13529.0, $P<0.001$.

Abbreviations: $\mathrm{HR}$, hazard ratio; $\mathrm{Cl}$, confidence interval; $\mathrm{CRRT}$, continuous renal replacement therapy; ECMO, extracorporeal membrane oxygenation; PTSD, posttraumatic stress disorder; BDZ, benzodiazepine.

risk of long-term mortality among sepsis survivors with sleep disorders was not much different from that in the general adult population of South Korea.

Additionally, the role of PTSD among survivors of sepsis might affect the results of this study. In a recent meta-analysis, the overall prevalence of PTSD symptoms among adult critical care survivors was $19.8 \% .{ }^{22}$ For sepsis patients, a prospective cohort study reported that $38 \%$ of patients reported PTSD symptoms in questionnaires after abdominal sepsis. ${ }^{23}$ PTSD among survivors of sepsis might have also caused the development of sleep disorders in our study, especially considering that disorders, such as insomnia and nightmares, are common in adults with PTSD symptoms. ${ }^{24}$ The prevalence of PTSD in sepsis survivors registered in the NHIS database was extremely low at $0.2 \%$; however, considering that PTSD might be accompanied by sleep disorder, ${ }^{17}$ many cases of PTSD among sepsis survivors were not captured in the NHIS database in this study.

Similarly, the results regarding sleep disorder with benzodiazepine or Z-drug are interesting. In South Korea, benzodiazepines and Z-drugs are cautiously prescribed by physicians to ensure that sepsis survivors with symptoms of severe sleep disorder continuously take these drugs. Additionally, another cohort study reported that benzodiazepine use was independently associated with a higher risk of mortality; ${ }^{25}$ thus, the pharmacologic effect of benzodiazepines might similarly affect the study results. However, information regarding this issue is still lacking, and more research is needed to confirm the relationship between sleep disorder, benzodiazepine or Z-drug use, and long-term survival among sepsis survivors. 
Table 4 Sensitivity Analyses of Multivariable Cox Regression Model for 5-Year All-Cause Mortality According to Sleep Disorder with Depression or PTSD and Sleep Disorder with BDZ or Z-Drug Use

\begin{tabular}{|c|c|c|}
\hline \multirow[t]{2}{*}{ Variable } & Multivariable Cox Model & \multirow[t]{2}{*}{$P$-value } \\
\hline & HR (95\% Cl) & \\
\hline \multicolumn{3}{|l|}{ Sleep disorder before and after sepsis (Model I) } \\
\hline Control group $(n=34,953)$ & I & \\
\hline Pre-sepsis sleep disorder group without PTSD or depression $(n=4796)$ & $1.19(1.13,1.25)$ & $<0.001$ \\
\hline Pre-sepsis sleep disorder group with PTSD or depression $(n=3 \mid 42)$ & $1.21(1.13,1.29)$ & $<0.001$ \\
\hline Post-sepsis sleep disorder group without PTSD or depression $(n=1878)$ & $1.67(1.56,1.79)$ & $<0.001$ \\
\hline Post-sepsis sleep disorder group with PTSD or depression $(n=1057)$ & $2.01(1.84,2.19)$ & $<0.001$ \\
\hline \multicolumn{3}{|l|}{ Sleep disorder before and after sepsis (Model 2) } \\
\hline Control group $(n=34,953)$ & 1 & \\
\hline Pre-sepsis sleep disorder group without BDZ or Z-drug use $(n=6409)$ & $1.18(1.13,1.23)$ & $<0.001$ \\
\hline Pre-sepsis sleep disorder group with BDZ or Z-drug use $(n=1529)$ & $1.30(1.18,1.43)$ & $<0.001$ \\
\hline Post-sepsis sleep disorder group without BDZ or Z-drug use $(n=2432)$ & $1.76(1.66,1.86)$ & $<0.001$ \\
\hline Post-sepsis sleep disorder group with BDZ or Z-drug use $(n=503)$ & $2.02(1.77,2.30)$ & $<0.001$ \\
\hline
\end{tabular}

Abbreviations: $\mathrm{HR}$, hazard ratio; $\mathrm{Cl}$, confidence interval; PTSD, post-traumatic stress disorder; BDZ, benzodiazepine.

The difference in the association of pre- and postsepsis sleep disorder groups with 5-year all-cause mortality is a notable finding in this study. A previous study reported that sleep disorders are highly comorbid with other critical medical conditions, such as diabetes mellitus, various cardiovascular diseases, respiratory, gastrointestinal, urinary, and neurologic disorders. ${ }^{26}$ Therefore, presepsis sleep disorder might reflect the comorbid medical status before sepsis treatment in this study. However, the post-sepsis sleep disorder diagnosis within 1 year after sepsis diagnosis might reflect sequelae from sepsis. As an important pathophysiology of sepsis, it has been reported that brain damage is caused by sepsis due to excessive microglial activation, impaired cerebral perfusion, and blood-brain-barrier dysfunction. ${ }^{27}$ Magnetic resonance imaging studies have shown that brain lesions and atrophy were dominant in sepsis patients with worse acute physiology and chronic health evaluation (APACHE) II and sequential organ failure assessment (SOFA) scores. $^{28}$ Since sleep disorders were common among patients with neurologic disorders, ${ }^{29}$ it is possible that the post-sepsis sleep disorder group has more sequelae than both the control and pre-sepsis sleep disorder groups, and this is attributable to the poorer long-term survival among sepsis survivors.

This study has several limitations. First, as we extracted and analyzed the data retrospectively, the reliability and quality of data might be limited. For example, we cannot guarantee that all sepsis survivors with sleep apnea (G47.3) underwent polysomnography for proper diagnosis in our study. Second, some important physiologic variables, such as body mass index, were not included in the analysis because they were not included in the NHIS database. Third, we used the ICD-10 codes registered in the NHIS database to calculate the Charlson comorbidity indexes of sepsis survivors. However, some of the underlying diseases specified using these ICD-10 codes might not reflect the actual underlying diseases. Fourth, we did not consider the type of sleep disorder such as insomnia and parasomnia. The different types of sleep disorders among sepsis survivors might affect the results of this study. Lastly, we did not include important patient parameters that may reflect the severity of sepsis. For example, the APACHE II and SOFA scores for sepsis patients were not included in our analysis because the NHIS database only provides data regarding the prescribed drugs, procedural information, and registered ICD-10 disease codes. However, we collected the data regarding endotracheal intubation, vasopressor, ventilator support, and CRRT and ECMO use during sepsis treatment to adjust for major organ failure in patients with sepsis.

\section{Conclusions}

In conclusion, $6.4 \%$ of sepsis survivors in South Korea were newly diagnosed with a sleep disorder within 1 year after 
sepsis diagnosis. Although both pre- and post-sepsis sleep disorders were associated with a higher 5-year all-cause mortality rate, the risk of 5-year all-cause mortality in the post-sepsis sleep disorder group was higher than that in the pre-sepsis sleep disorder group. In addition, these association was more evident in the sleep disorder group with PTSD or depression and benzodiazepine or Z-drug user. Our results suggest that sepsis survivors who experience sleep disorder before and after sepsis constituted the highrisk group, towards which interventions may be directed.

\section{Abbreviations}

APACHE, acute physiology and chronic health evaluation; $\mathrm{CI}$, confidence interval; CRRT, continuous renal replacement; ECMO, extracorporeal membrane oxygenation; HR, hazard ratio; ICD-10, International Classification of Diseases, 10th revision; ICU, intensive care units; NHIS, National Health Insurance Service; PTSD, post-traumatic stress disorder; SOFA, sequential organ failure assessment.

\section{Data Sharing Statement}

All data will be available upon reasonable request to corresponding author.

\section{Ethics Approval and Consent to Participate}

The study protocol was approved by the Institutional Review Board of Seoul National University Bundang Hospital (X-1912-580-902) and the Health Insurance Review and Assessment Service (NHIS-2020-1-095). The requirement for informed consent was waived because the data analyses were performed retrospectively using anonymized data derived from the South Korean NHIS database.

\section{Acknowledgments}

In-Ae Song and Hye Yoon Park are co-first authors for this study.

\section{Author Contributions}

Tak Kyu Oh designed the study, analysed the data, interpreted the data, and drafted the manuscript; Hye Yoon Park and In-Ae Song contributed to the study conceptualization, acquisition of data, and review of manuscript.; All authors contributed to data analysis, drafting or revising the article, have agreed on the journal to which the article will be submitted, gave final approval of the version to be published, and agree to be accountable for all aspects of the work.

\section{Funding}

This research did not receive any specific grant from funding agencies in the public, commercial, or not-forprofit sectors.

\section{Disclosure}

The authors declare that they have no competing interests.

\section{References}

1. Shankar-Hari M, Phillips GS, Levy ML, et al. Developing a new definition and assessing new clinical criteria for septic shock: for the Third International Consensus Definitions for Sepsis and Septic Shock (Sepsis-3). JAMA. 2016;315(8):775-787. doi:10.1001/ jama.2016.0289

2. Rhee C, Dantes R, Epstein L, et al. Incidence and trends of sepsis in US hospitals using clinical vs claims data, 2009-2014. JAMA. 2017;318(13):1241-1249. doi:10.1001/jama.2017.13836

3. Meyer N, Harhay MO, Small DS, et al. Temporal trends in incidence, sepsis-related mortality, and hospital-based acute care after sepsis. Crit Care Med. 2018;46(3):354-360. doi:10.1097/CCM.00000000 00002872

4. Shankar-Hari M, Harrison DA, Rubenfeld GD, Rowan K. Epidemiology of sepsis and septic shock in critical care units: comparison between sepsis-2 and sepsis-3 populations using a national critical care database. $B r \quad J$ Anaesth. 2017;119(4):626-636. doi:10.1093/bja/aex234

5. Rhodes A, Evans LE, Alhazzani W, et al. Surviving sepsis campaign: international Guidelines for Management of Sepsis and Septic Shock: 2016. Intensive Care Med. 2017;43(3):304-377.

6. Kaukonen KM, Bailey M, Suzuki S, Pilcher D, Bellomo R. Mortality related to severe sepsis and septic shock among critically ill patients in Australia and New Zealand, 2000-2012. JAMA. 2014;311 (13):1308-1316. doi:10.1001/jama.2014.2637

7. Milano PK, Desai SA, Eiting EA, Hofmann EF, Lam CN, Menchine M. Sepsis bundle adherence is associated with improved survival in severe sepsis or septic shock. West J Emerg Med. 2018;19 (5):774-781. doi:10.5811/westjem.2018.7.37651

8. Fleischmann-Struzek C, Mikolajetz A, Schwarzkopf D, et al Challenges in assessing the burden of sepsis and understanding the inequalities of sepsis outcomes between National Health Systems: secular trends in sepsis and infection incidence and mortality in Germany. Intensive Care Med. 2018;44(11):1826-1835. doi:10.1007/s00134-018-5377-4

9. Pavlova MK, Latreille V. Sleep disorders. Am J Med. 2019;132 (3):292-299. doi:10.1016/j.amjmed.2018.09.021

10. Alexander M, Ray MA, Hebert JR, et al. The National Veteran Sleep Disorder Study: descriptive Epidemiology and Secular Trends, 20002010. Sleep. 2016;39(7):1399-1410. doi:10.5665/sleep.5972

11. Huyett P, Siegel N, Bhattacharyya N. Prevalence of Sleep Disorders and Association With Mortality: results from the NHANES 20092010. Laryngoscope. 2020. doi:10.1002/lary.28900

12. Trompeo AC, Vidi Y, Locane MD, et al. Sleep disturbances in the critically ill patients: role of delirium and sedative agents. Minerva Anestesiol. 2011;77(6):604-612.

13. Eddleston JM, White P, Guthrie E. Survival, morbidity, and quality of life after discharge from intensive care. Crit Care Med. 2000;28 (7):2293-2299. doi:10.1097/00003246-200007000-00018 
14. Rundshagen I, Schnabel K, Wegner C, Am Esch SJ. Incidence of recall, nightmares, and hallucinations during analgosedation in intensive care. Intensive Care Med. 2002;28(1):38-43. doi:10.1007/ s00134-001-1168-3

15. Jones C, Griffiths RD, Humphris G, Skirrow PM. Memory, delusions, and the development of acute posttraumatic stress disorder-related symptoms after intensive care. Crit Care Med. 2001;29(3):573-580. doi:10.1097/00003246-200103000-00019

16. Vandeputte M, de Weerd A. Sleep disorders and depressive feelings: a global survey with the Beck depression scale. Sleep Med. 2003;4 (4):343-345. doi:10.1016/s1389-9457(03)00059-5

17. Harvey AG, Jones C, Schmidt DA. Sleep and posttraumatic stress disorder: a review. Clin Psychol Rev. 2003;23(3):377-407. doi:10.1016/s0272-7358(03)00032-1

18. Oh TK, Park HY, Song IA. Benzodiazepine use and long-term mortality in South Korean Adult Population: a Cohort Study. Yonsei Med J. 2021;62(6):528-534. doi:10.3349/ymj.2021.62.6.528

19. Huang CY, Daniels R, Lembo A, et al. Life after sepsis: an international survey of survivors to understand the post-sepsis syndrome. Int J Qual Health Care. 2019;31(3):191-198. doi:10.1093/intqhe/ mzy 137

20. Altman MT, Knauert MP, Pisani MA. Sleep disturbance after hospitalization and critical illness: a Systematic Review. Ann Am Thorac Soc. 2017;14(9):1457-1468. doi:10.1513/AnnalsATS.201702-148SR

21. Oh TK, Song I-A. Five-year mortality trends associated with sleep disorders in South Korea: a population-based cohort study. Sleep Biol Rhythms. 2021:1-8.

22. Righy C, Rosa RG, da Silva RTA, et al. Prevalence of post-traumatic stress disorder symptoms in adult critical care survivors: a systematic review and meta-analysis. Crit Care. 2019;23(1):213. doi:10.1186/ s13054-019-2489-3
23. Boer KR, van Ruler O, van Emmerik AA, et al. Factors associated with posttraumatic stress symptoms in a prospective cohort of patients after abdominal sepsis: a nomogram. Intensive Care Med. 2008;34(4):664-674. doi:10.1007/s00134-007-0941-3

24. Koffel E, Khawaja IS, Germain A. Sleep disturbances in posttraumatic stress disorder: updated review and implications for treatment. Psychiatr Ann. 2016;46(3):173-176. doi:10.3928/0048571320160125-01

25. Patorno E, Glynn RJ, Levin R, Lee MP, Huybrechts KF. Benzodiazepines and risk of all cause mortality in adults: cohort study. BMJ. 2017;358:j2941. doi:10.1136/bmj.j2941

26. Dikeos D, Georgantopoulos G. Medical comorbidity of sleep disorders. Curr Opin Psychiatry. 2011;24(4):346-354. doi:10.1097/ YCO.0b013e3283473375

27. Sonneville R, Verdonk F, Rauturier C, et al. Understanding brain dysfunction in sepsis. Ann Intensive Care. 2013;3(1):15. doi:10.1186/2110-5820-3-15

28. Orhun G, Esen F, Ozcan PE, et al. Neuroimaging findings in sepsis-induced brain dysfunction: association with clinical and laboratory findings. Neurocrit Care. 2019;30(1):106-117. doi:10.1007/s12028-018-0581-1

29. Ju YS, Videnovic A, Vaughn BV. Comorbid sleep disturbances in neurologic disorders. Continuum (Minneap Minn). 2017;23(4, Sleep Neurology):1117-1131. doi:10.1212/CON.0000000000000501
Nature and Science of Sleep

\section{Publish your work in this journal}

Nature and Science of Sleep is an international, peer-reviewed, open access journal covering all aspects of sleep science and sleep medicine, including the neurophysiology and functions of sleep, the genetics of sleep, sleep and society, biological rhythms, dreaming, sleep disorders and therapy, and strategies to optimize healthy sleep.

\section{Dovepress}

The manuscript management system is completely online and includes a very quick and fair peer-review system, which is all easy to use. Visit http://www.dovepress.com/testimonials.php to read real quotes from published authors. 